vitamin E every other day or placebo and $500 \mathrm{mg}$ vitamin $\mathrm{C}$ daily or placebo. During the study (mean follow-up 8 years), 1,661 deaths occurred, 509 of which had a cardiovascular cause; 511 men experienced a myocardial infarction and 464 had a stroke. The rate of major cardiovascular events did not differ significantly between vitamin $\mathrm{C}$ and placebo or between vitamin $\mathrm{E}$ and placebo (10.8 versus 10.9 per 1,000 person years, respectively for both comparisons). No significant effect was observed on the risk of individual end points (myocardial infarction, stroke, cardiovascular mortality, and all-cause mortality) for either vitamin $\mathrm{C}$ or vitamin $\mathrm{E}$ when compared with placebo. Vitamin E was, however, associated with an increase in the risk of hemorrhagic stroke. No significant difference existed in the number of adverse events between placebo and either of the vitamin supplements. The results of this trial do not support the use of vitamins $\mathrm{C}$ or $\mathrm{E}$ supplements for the prevention of cardiovascular events in men.

Original article Sesso HD et al. (2008) Vitamins E and C in the prevention of cardiovascular disease in men. The Physician's Health Study II randomized controlled trial. JAMA 300: 2123-2133

\section{Chlorthalidone is best for preventing heart failure in patients with hypertension}

Davis et al. have published findings from the Antihypertensive and Lipid-Lowering Treatment to Prevent Heart Attack Trial (ALLHAT) demonstrating that chlorthalidone is superior to both amlodipine and doxazosin for the prevention of new-onset heart failure (HF) with preserved $(>50 \%)$ and reduced $(<50 \%)$ ejection fraction in patients with hypertension.

In this randomized, double-blind, multicenter trial, ejection fraction data were available for 910 of 1,367 patients hospitalized with HF. All participants were aged 55 years or older, had hypertension and one additional cardiovascular risk factor, and were randomly assigned to treatment with chlorthalidone, amlodipine, lisinopril, or doxazosin. Hospitalization for HF occurred with preserved and reduced ejection fraction in $44 \%$ and $56 \%$ of patients, respectively. HF-related mortality was higher among patients with reduced ejection fraction compared with those who had preserved ejection fraction $(41.9 \%$ versus $29.2 \%, P<0.001)$. Chlorthalidone was associated with a $30-48 \%$ reduction in the risk of $\mathrm{HF}$ with preserved ejection fraction compared with the other study drugs. The risk of HF with reduced ejection fraction was lower among patients who received chlorthalidone than in those on amlodipine (by 22\%) or doxazosin (by $36 \%)$, but no difference in risk reduction was found between the chlorthalidone and lisinopril arms. The authors postulate that a combination of chlorthalidone and lisinopril would be most effective for the prevention of HF in older patients with hypertension.

\footnotetext{
Original article Davis BR et al. for the ALLHAT

Collaborative Research Group (2008) Heart failure with preserved and reduced left ventricular ejection fraction in the Antihypertensive and Lipid-Lowering treatment to prevent Heart Attack Trial. Circulation 118: 2259-2267
} 